\title{
Vitamin B2 Intake and the Risk of Colorectal Cancer: a Meta- Analysis of Observational Studies
}

\author{
Yan Liu' ${ }^{1}$, Qiu-Yan Yu ${ }^{1}$, Zhen-Li Zhu ${ }^{1}$, Ping-Yi Tang ${ }^{2}, K_{e}$ Li $^{1 *}$
}

\begin{abstract}
Background: A systematic review and meta-analysis of observational studies evaluated the association of intake of vitamin B2 with the incidence of colorectal cancer. Materials and Methods: Relevant studies were identified in MEDLINE via PubMed (published up to April 2014). We extracted data from articles on vitamin $B 2$ and used multivariable-adjusted odds ratio (OR) and a random-effects model for analysis. Results: We found 8 articles meeting the inclusion criteria (4 of cohort studies and 4 of case-control studies) and a total of 7,750 colorectal cancer cases were included in this meta-analysis. The multivariable-adjusted OR for pooled studies for the association of the highest versus lowest vitamin $\mathrm{B} 2$ intake and the risk of colorectal cancer was $0.83(95 \%$ confidence interval $[95 \% \mathrm{CI}]: 0.75,0.91)$. We performed a sensitivity analysis for vitamin $\mathrm{B} 2$. If we omitted the study by Vecchia et al., the pooled OR was $0.86(95 \% \mathrm{CI}, 0.77,0.96)$. Conclusions: This is the first meta-analysis to study links between vitamin $B 2$ and colorectal cancer. We found vitamin B2 intake was inversely associated with risk of colorectal cancer. However, further research and large sample studies need to be conducted to better validate the result.
\end{abstract}

Keywords: Colorectal cancer - vitamin B2 - incidence - observational study - meta-analyses

Asian Pac J Cancer Prev, 16 (3), 909-913

\section{Introduction}

In 2013, colorectal cancer was newly diagnosed in 73,680 males in the United States; 26,300 died of the disease. It was newly diagnosed in 69,140 females and 24,530 died (Siegel et al., 2013). Indeed, colorectal cancer is the third most commonly diagnosed cancer in males and the second in females, with more than 1.2 million new cases and 608,700 deaths estimated to have occurred in 2008 (Center et al., 2009).

Vitamin B2 (chemical formula: C17H20N4O6) also known as riboflavin. In fact, the flavin in riboflavin comes from flavus, the Latin word for yellow. (yellow enzymes play a role of hydrogen in the biological oxidation reduction). Vitamin B2 is one of many nutrients required to recycle glutathione, which is one of the most important antioxidants in the human body. (From a chemical standpoint, what B2 does is facilitate the conversion of oxidized glutathione into reduced glutathione.) (Yasuda and Hiraoka, 1999).

Riboflavin is unique among the water-soluble vitamins in that milk and dairy products make the greatest contribution to its intake in Western diets. Meat and fish are also good sources of riboflavin, and certain fruit and vegetables, especially dark-green vegetables, contain reasonably high concentrations (Powers, 2003). Vitamin B2 has limited storage, So it need a food supplement every day.

The risk of deficiency of vitamin B2: it will affect the body's biological oxidation and make the metabolic disorder (Christensen, 1993). Vitamin B2 deficiency include cracking of the lips and corners of the mouth, an inflamed tongue, loss of visual perception and sensitivity to light, cataracts, and burning and/or itching of the eyes, lips, mouth, and tongue. It may also result in dull or oily hair, an oily skin, premature wrinkles on the face and arms, and split nails (Roe, 1991).

Other new functions of vitamin B2 are treatment of migraine, cardiovascular diseases and a prevention against cancer (Podorozhnyi et al., 1990; Powers, 2003). RCT studies in adults has confirmed that riboflavin is safe and probably effective in migraine prophylaxis, based on level B evidence. The bridge is troubled mitochondria, Riboflavin, a major co-factor in oxidative metabolism, may overcome this impairment (Colombo et al., 2014). Riboflavin may be a preventative medication for paediatric migraine (O'Brien and Hershey, 2010). Riboflavin plays a protective role in ischemia-reperfusion injury. Some experiments about blood biomarkers related to status of vitamin have been done in healthy women and cardiovascular patients (Midttun et al., 2014). Epidemiological studies have proved low folate intake with an increased risk of epithelial cancers, including colorectal cancer and cervical cancer. Riboflavin has 
received much less attention, but there is increasing interest in the well-established role that flavins play in folate metabolism and the possible synergy of a protective effect between these 2 vitamins for cancer (Powers, 2005).

Our article mainly used a systematic review and meta-analysis of observational studies assessing the association of intake of vitamin B2 and the incidence of colorectal cancer. This is the first meta-analysis to study the relationship between vitamin $\mathrm{B} 2$ and colorectal cancer. Through our articles, which will provide the scientific basis for future prevention and treatment of colorectal cancer.

\section{Materials and Methods}

We performed a literature search of MEDLINE via Pubmed to identify observational studies of colorectal cancer published up to April 2014, without any language restrictions. We used the following $\mathrm{MeSH}$ terms and free text words: 1. (colorectal OR colorectum OR colon OR colonic OR rectal OR rectum) AND (neoplasm OR cancer OR adenocarcinoma OR carcinoma OR tumour OR tumor). 2. Vitamin OR vitamin supplement OR retinol OR retinal OR thiamine OR cobalamin OR riboflavin OR niacin OR nicotinic acid OR pantothenic acid OR pyridoxine OR biotin OR folic acid OR folate OR ascorbic acid OR bravery calcitonin OR tocopherol OR phylloquinone OR menadione. 3. "Cohort Studies"[Mesh]) OR "Follow-Up Studies"[Mesh] OR "Prospective Studies"[Mesh] OR "Case-Control Studies"[Mesh]. In addition, we searched reference lists of retrieved reviews or meta-analyses for studies (Stroup et al., 2000).

\section{Eligibility criteria}

Studies were included if (1) they were observational studies; (2) the exposure of interest was vitamin B2 intake; (3) the outcome of interest was the incidence of colorectal, colon, or rectal cancer; (4) relative risk (RR) or odds ratio (OR) estimates with $95 \%$ confidence intervals (95\%CIs) were reported; and the (5) Newcastle-Ottawa Scale (NOS) quality grade for observational studies in meta-analyses (Wells et al., 2000) was $>6$. With multiple publication of the same study or similar documents, we retained the article with the best quality. We did not include reviews or meta-analyses.

Three investigators reviewed all eligible studies and extracted in duplicate the following data: the first author's last name, publication year; country where the study was performed; sex and age of subjects; sample size (cases and controls or cohort size); measurement method; range of exposure; NOS grade; variables adjusted in the analyses; and RR or OR estimates with corresponding 95\%CIs for the highest versus lowest categories of intake. If results of the study were reported several times, we chose the best quality study. If data collected were not reported in the primary article, items were treated as "not reported." We did not contact the author for the information.

For each eligible study, two reviewers (Y.L and Q.Y) independently performed a quality assessment in duplicate by using the NOS. The scale is divided into 3 parts -- "selection", "comparability", "outcome" -- for cohort study, for a possible total of 9 stars. Articles with $<6$ stars were excluded.

\section{Statistical analysis}

Study-specific OR estimates were combined by use of a random-effects model, which considers both within- and between-study effects (DerSimonian and Laird, 1986). If results were reported for both dietary and total vitamins (food and supplements combined), we used the results for total vitamins in the main analysis (Zhang et al., 2006). If results were reported for both men and women and both, we used the results for both in the main analysis.

Commonly, 3 to 5 grades are reported for vitamin intake. We used the results (RR or OR) for the highest versus lowest categories. In addition, if the article gave different times (e.g., > 5 years; $>10$ years; $>15$ years) for vitamin intake and RR, we used the RR corresponding to the longest time because vitamin intake needs a long time to affect the incidence of colorectal cancer.

We used the random-effects model to incorporate the different studies (DerSimonian and Laird, 1986). When the incidence of disease is very low, in epidemiological studies, we can combine cohort studies and the results of the case-control studies, and use the OR approximate value instead of the RR (Greenland, 1987). Statistical heterogeneity was evaluated by the inconsistency statistic $\left(\mathrm{I}^{2}\right)$. In 2011, the Cochrane Collaboration published updated information on the $\mathrm{I}^{2}$ (JPT and Green, 2011). There are 4 degrees for heterogeneity; with $\mathrm{I}^{2}<50 \%$, we considered the heterogeneity acceptable. With $\mathrm{I}^{2} \geq 50 \%$, we performed sensitivity and stratified analyses.

The combined OR were estimated from forest plots (Peters et al., 2008). We performed a sensitivity analysis removing one study at a time and analyzing the remaining studies to evaluate the effect of a single study. Publication bias was evaluated by the Begg and Egger regression asymmetry tests (Song and Gilbody, 1998). If the article showed publication bias, we used the trim and fill method (Duval and Tweedie, 2000a). Statistical analysis involved use of Stata v12.0 (Stata Corp, College Station, TX). $p<0.05$ was considered statistically significant.

\section{Results}

\section{Literature search}

The details of the literature search are in Figure 1. Briefly, we reviewed 9 articles of vitamin B2 intake and risk of colorectal cancer (La Vecchia et al., 1997; Jedrychowski et al., 2001; Shin et al., 2006; de Vogel et al., 2008; E Ma et al., 2009; Shrubsole et al., 2009; van Lee et al., 2011; Bassett et al., 2013; Zschabitz et al., 2013), published from 1997 to 2013. But we found the article has been published by Shin in 2006 and the article has been published by Shrubsole in 2009 are using the same cohort: The Shanghai Women's Health Study (SWHS). If results of the study were reported several times, we chose the best quality study. So we chose the article has been published by Shrubsole in 2009. There are 5 cohort studies and 4 case-control studies in total. One cohort study conducted in Netherlands, the multivariable-adjusted RR of women and men were separate, so we regarded the study as 2 


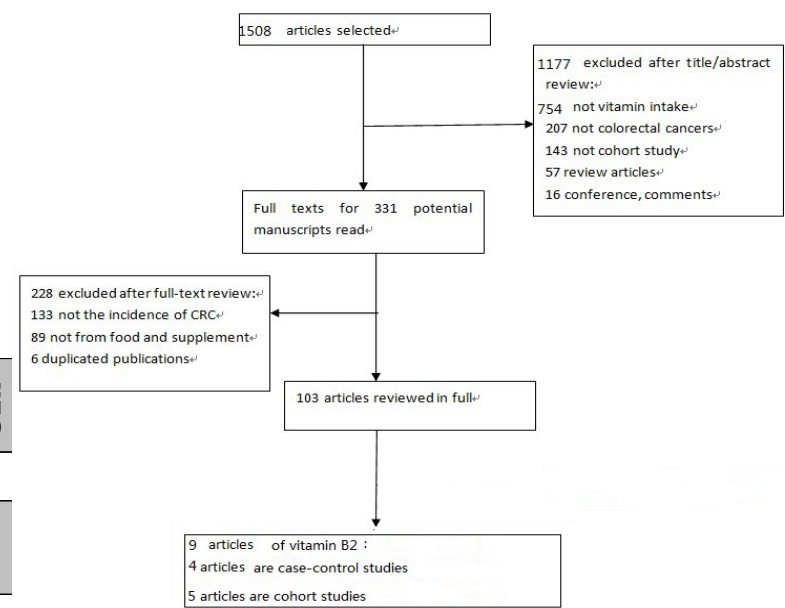

Figure 1. Flowchart of the Selection of Articles

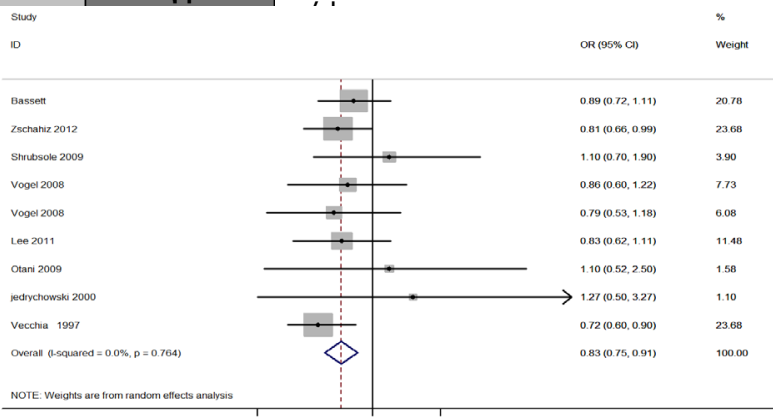

Figure 2. The Forest Plot for Vitamin B2 the Odds

Ratio (OR); 95\% Confidence Interval (CI); and

Relative Weight are Show; $\mathrm{I}^{2}$, index of Heterogeneity

cohort studies.

Study characteristics

Characteristics of included studies are summarized in Table 1. The 8 vitamin B2 (riboflavin) articles (7750 cases, age range 40-79 years) were published between 1997 and 2013; 2were conducted in the Australia and 1 each in USA, Japan, Italy, Poland, Netherlands and China. The subjects of 2 studies were female and subjects of 6 were both female and male. Studies of Vitamin B2 intake met the quality criteria (6-8 stars): 1 article had 8 stars, 6 had 7 stars and 1 had 6 stars.

Association of vitamin intake and colorectal cancer

The multivariable-adjusted OR for each study and all studies combined for the highest versus lowest categories of intake of vitamins B2 (riboflavin) is in Figure 2. The association of vitamin B2 (riboflavin) and colorectal cancer was significant, with the heterogeneity $\left(\mathrm{I}^{2}=0.0 \%\right)$. The pooled OR for the highest versus lowest categories of vitamin B2 (riboflavin) intake and colorectal cancer risk was 0.83 (95\% CI, 0.75-0.91).

\section{Sensitivity analyses}

Sensitivity analysis is used to study the stability of the results after removed a study (JPT and Green, 2011). We performed a sensitivity analysis for vitamin B2. If we omitted the study by Vecchia et al., the pooled OR was 0.86 (95\%CI, 0.77,0.96).

\section{Publication bias}

Begg and Egger tests were used to examine publication bias 
(Begg and Mazumdar, 1994). With the Egger test (Higgins, 2008), we found some publication bias for vitamins B2 (riboflavin) ( $p=0.039)$, but the result of Begg's Test is $p=0.076$. So the Egger tests seems to be more sensitive. We used the trim and fill method (Duval and Tweedie, 2000b) for vitamin B2 findings and found a pooled OR of 0.81 (95\%CI, 0.73-0.89), which before the adjustment was 0.83 (95\%CI, 0.75-0.91). But, when the study exist the high weight worth and extremely positive result, then the trim and fill method could not do about it. When the studies were less than 10 research, we do not recommend map funnel and tested for the symmetry of funnel plot, because too little studies including in research, the power of the test is low, which is not enough to find funnel plot is truly asymmetry. So our analyses about publication bias and the trim and fill method is just to provide a reference for everyone, not to make a conclusion.

\section{Discussion}

This is the first meta-analysis evaluating the relationship between vitamin B2 intake and colorectal cancer. In this meta-analysis of epidemiological studies including 5 cohort studies and 4 case-control studies, we found vitamin B2 intake had an inverse association with the incidence risk of colorectal cancer.

If we separate the cohort studies and case-control studies to analysis, the OR of cohort studies is 0.86 (95\% CI, $0.76,0.97)$ and $\mathrm{I}^{2}$ is $0.0 \%$. The OR of case-control studies is $0.78(95 \% \mathrm{CI}, 0.66,0.91)$ and $\mathrm{I}^{2}$ is $0.0 \%$. The combination of cohort studies and case-control studies is $0.83(95 \% \mathrm{CI}, 0.75-0.91)$ and $\mathrm{I}^{2}$ is $0.0 \%$. So the results of separate and combined analysis are consistent. No heterogeneity were detected in cohort studies , casecontrol studies and the combination of cohort studies and case-control studies. We performed a sensitivity analysis for the result of combination cohort studies and casecontrol studies after we omitted the study by Vecchia et al., the pooled OR was $0.86(95 \% \mathrm{CI}, 0.77,0.96)$. So our result is relatively reliable.

There are ten studies after reviewing all articles, but except multiple publication of the same study or similar documents, only 9 studies are in this meta analyses. So the if studies were less than 10, The funnel plot and tested for the symmetry of funnel plot are not appropriate, the power of the test is low when studies were less than 10 , which is not enough to find funnel plot is truly asymmetry (Nolting et al., 2012). We used both Begg and Egger tests to examine publication bias. the Egger tests seems to be more sensitive. With the Egger test, we found some publication bias for vitamins B2 (riboflavin) $(p=0.039)$, but the result of Begg's Test is $p=0.076$ seemed to be no publication bias. So our result about publication may be not Correct or accurate. In other words, perhaps there is no need to test the publication bias.

Some studies about the hypermethylation of the human riboflavin transporter 2 (hRFT2) gene and cervical squamous cell carcinoma (CSCC) patients have been done (Ma et al., 2014). Some studies about antitumor activity of irradiated riboflavin on human renal carcinoma cell line 786-O show the potential of riboflavin for treating cancer (Chaves et al., 2014). In breast cancer, a significant decrease in riboflavin serum levels and increase in riboflavin carrier protein occurs, indicating a potential role of riboflavin in disease progression (Bareford, et al., ww2013). Some findings suggested that riboflavin at high doses might promote lung cancer progression (Yang et al., 2013). Defective expression of C20orf54 gene statuses is associated with the development of esophageal squamous cell carcinoma and this may represent a mechanism underlying the decreased plasma riboflavin levels in ESCC (Ainiwaer et al., 2013). Decreased blood riboflavin levels are correlated with defective expression of RFT2 gene in gastric cancer (Eli et al., 2012). But, there are not studies about riboflavin and colorectal cancer. So our study will fill the void and provided some evidence to prevent colorectal cancer.

This meta-analysis has several strengths. First, this is the first meta-analysis evaluating the relationship between vitamin B2 intake and colorectal cancer. We found vitamin B2 intake had an inverse association with the incidence risk of colorectal cancer. Second, the result of cohort studies, case-control studies and the result of combination are consistent. Additional strengths are the extensive search of the literature and examination of the retrieved materials by at least two reviewers and Strict evaluation standard .

Our study has several limitations. First, as a meta analysis of observational data, our results had recall and selection bias inherent in the original studies. Second, the adjusted factors differed in many studies and commonly included age, sex, energy, ethnicity, body mass index, physical activity, pack-years of smoking, family history of colorectal cancer, colorectal cancer screening, and intake of processed meat and alcohol. Finally, we used pooled RRs for the highest versus lowest doses, but the definite dose differed slightly among studies. Given the limitation of this study, larger studies with a prospective design should be considered and investigated to validate the current findings.

In conclusion, a meta-analysis suggested that higher intake of vitamins B2 (riboflavin) could reduce the incidence risk of colorectal cancer. Further researches and large sample studies need to be conducted to better validate the potentially protective mechanism in vitamins B2 (riboflavin) and the relationship between vitamins B2 (riboflavin) intake and the risk of colorectal cancer..

\section{References}

Ainiwaer J, Tuerhong A, Hasim A, et al (2013). Association of the plasma riboflavin levels and riboflavin transporter (C20orf54) gene statuses in Kazak esophageal squamous cell carcinoma patients. Mol Biol Rep 40, 3769-75.

Bareford L M, Avaritt B R, Ghandehari H, Nan A, Swaan P W (2013). Riboflavin-targeted polymer conjugates for breast tumor delivery. Pharm Res, 30, 1799-812.

Bassett J K, Severi G, Hodge A M, et al (2013). Dietary intake of $\mathrm{B}$ vitamins and methionine and colorectal cancer risk. Nutr Cancer, 65, 659-67.

Begg C B, and Mazumdar M (1994). Operating characteristics of a rank correlation test for publication bias. Biometrics, 1088-101 
Center M M, Jemal A, Smith R A, Ward E (2009). Worldwide variations in colorectal cancer. CA: Cancer J Clin, 59, 366-78

Chaves Neto A H, Pelizzaro-Rocha K J, Fernandes M N, FerreiraHalder C V (2014). Antitumor activity of irradiated riboflavin on human renal carcinoma cell line 786-O. Tumour Biol.

Christensen H. N. (1993). Riboflavin can protect tissue from oxidative injury. Nutr Rev, 51, 149-150.

Colombo B, Saraceno L, Comi G (2014). Riboflavin and migraine: the bridge over troubled mitochondria. Neurol Sci, 35, 141-4.

de Vogel S, Dindore V, van Engeland M, et al (2008). Dietary folate methionine riboflavin and vitamin B-6 and risk of sporadic colorectal cancer. $J$ Nutr, 138, 2372-8.

DerSimonian R, Laird N (1986). Meta-analysis in clinical trials. Controlled clinical trials, 7, 177-88.

Duval S, Tweedie R (2000a). A nonparametric "trim and fill" method of accounting for publication bias in meta-analysis. $J$ Am Statistical Association, 95, 89-98.

Duval S, Tweedie R. (2000b). Trim and fill: A simple funnel plot-based method of testing and adjusting for publication bias in meta analysis. Biometrics, 56, 455-463.

Eli M, Li DS, Zhang WW, et al (2012). Decreased blood riboflavin levels are correlated with defective expression of RFT2 gene in gastric cancer. World J Gastroenterol, 18, 3112-8.

Greenland S (1987). Quantitative methods in the review of epidemiologic literature. Epidemiol Rev, 9, 1-30

Higgins J (2008). Green S. Cochrane handbook for systematic reviews of interventions version 5.1.0.

Jedrychowski W, Steindorf K, Popiela T, et al (2001). Risk of colorectal cancer from alcohol consumption at lower vitamin intakes. A hospital-based case-control study in Poland. Rev Environ Health, 16, 213-222

JPT CHH, Green S (2011). Cochrane handbook for systematic reviews of interventions version 5.1. 0 [updated March 2011]. The Cochrane Collaboration.

La Vecchia C, Braga C, Negri E, et al (1997). Intake of selected micronutrients and risk of colorectal cancer. Int J Cancer, 73, 525-30

Ma E, Iwasaki M, Kobayashi M, et al (2009). Dietary intake of folate vitamin B2 vitamin B6 vitamin B12 genetic polymorphism of related enzymes and risk of breast cancer: a case-control study in Japan. Nutr Cancer, 61, 447-456.

Ma JQ, Kurban S, Zhao JD, Li QZ, Hasimu A (2014). Epigenetic regulation of human riboflavin transporter 2 (hRFT2) in cervical cancers from Uighur women. Asian Pac J Cancer Prev, 15, 2485-9

Midttun O, Townsend MK, Nygard O, et al (2014). Most blood biomarkers related to vitamin status one-carbon metabolism and the kynurenine pathway show adequate preanalytical stability and within-person reproducibility to allow assessment of exposure or nutritional status in healthy women and cardiovascular patients. J Nutr, 144, 784-790.

Nolting A, Perleth M, Langer G, et al (2012). [GRADE guidelines: 5 . Rating the quality of evidence: publication bias]. Z Evid Fortbild Qual Gesundhwes, 106, 670-6.

O'Brien HL, Hershey AD (2010). Vitamins and paediatric migraine: Riboflavin as a preventative medication. Cephalalgia, 30, 1417-18.

Peters JL, Sutton AJ, Jones DR, Abrams KR, Rushton L (2008). Contour-enhanced meta-analysis funnel plots help distinguish publication bias from other causes of asymmetry. J Clin Epidemiol, 61, 991-6

Podorozhnyi PG, Vdovichenko VI, Rozanov EM, et al (1990). Vitamin deficiency in patients with ischemic heart disease. Vrach Delo, 9, 18-19 (in Russian).
Powers HJ (2003). Riboflavin (vitamin B-2) and health. Am J Clin Nutr, 77, 1352-60

Powers HJ (2005). Interaction among folate riboflavin genotype and cancer with reference to colorectal and cervical cancer. J Nutr, 135, 2960-66

Roe DA (1991). Riboflavin deficiency: mucocutaneous signs of acute and chronic deficiency. Semin Dermatol, 10, 293-5

Shin A, Li H, Shu XO, et al (2006). Dietary intake of calcium fiber and other micronutrients in relation to colorectal cancer risk: Results from the Shanghai Women's Health Study. Int J Cancer, 119, 2938-42.

Shrubsole MJ, Yang G, Gao YT, et al (2009). Dietary B vitamin and methionine intakes and plasma folate are not associated with colorectal cancer risk in Chinese women. Cancer Epidemiol Biomarkers Prev, 18, 1003-6.

Siegel R, Naishadham D, Jemal A (2013). Cancer statistics 2013. CA: Cancer J Clin, 63, 11-30

Song F, Gilbody S. (1998). Bias in meta-analysis detected by a simple graphical test. Increase in studies of publication bias coincided with increasing use of meta-analysis. $B M J$, 316, 471 .

Stroup DF, Berlin JA, Morton SC, et al (2000). Meta-analysis of observational studies in epidemiology: a proposal for reporting. JAMA, 283, 2008-12

van Lee L, Heyworth J, McNaughton S, et al (2011). Selected dietary micronutrients and the risk of right- and left-sided colorectal cancers: a case-control study in Western Australia. Ann Epidemiol, 21, 170-7.

Wells G, Shea B, O'connell D, et al (2000). The Newcastle-Ottawa Scale (NOS) for assessing the quality of nonrandomised studies in meta-analyses.

Yang HT, Chao PC, Yin MC (2013). Riboflavin at high doses enhances lung cancer cell proliferation invasion and migration. J Food Sci, 78, 343-9.

Yasuda K, Hiraoka M. (1999). [Vitamin B2 (riboflavin)]. Nihon Rinsho, 57, 128-30.

Zhang SM, Moore SC, Lin J, et al (2006). Folate vitamin B6 multivitamin supplements and colorectal cancer risk in women. Am J Epidemiol, 163, 108-115

Zschabitz S, Cheng TY, Neuhouser ML, et al (2013). B vitamin intakes and incidence of colorectal cancer: results from the Women's Health Initiative Observational Study cohort. Am J Clin Nutr, 97, 332-43. 